\title{
АВТОМАТИЗАЦИЯ МОДЕЛИРОВАНИЯ И ПРОГНОЗИРОВАНИЯ АТМОСФЕРНОГО ЗАГРЯЗНЕНИЯ
}

\author{
Сулейманов Т.И. ${ }^{1}$, Мустафазаде Н.Х. ${ }^{2}$, Гулузаде Р.К. ${ }^{3}$, Гаджиев М.М. ${ }^{4}$, Иванова Л.В. \\ 1,2,3 - Национальное Аэрокосмическое Агентство, Азербайджанская Республика. \\ 4 - Одесская национальная академия связи им. А.С. Попова, Украина \\ 5 - Одесский технический колледж Одесской национальной академии пищевых технологий, Украина \\ ORCID: ${ }^{4}$ http://orcid.org/0000-0001-7280-3863, ${ }^{5}$ http://orcid.org/0000-0003-1738-7697 \\ E-mail:_
}

Copyright (C) 2018 by author and the journal “Automation technologies and business - processes. This work is licensed under the Creative Commons Attribution International License (CC BY). http://creativecommons.org/licanses/by/4.0

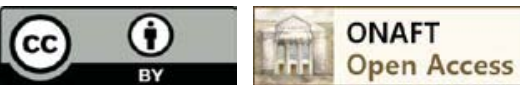

DOI:

Аннотация: В работе описана новая программа для автоматизачии обработки данных мониторинга, моделирования и прогнозирования атмосферного загрязнения. Предложена модель системы мониторинга атмосферного загрязнения, которая позволяет выразить зависимость конщентрации выбранного загрязнителя атмосферы от совокупности метеорологических факторов. Модель строится по методу группового учета аргументов, хорошо зарекомендовавшему себя при многофакторном моделировании. Построение модели осуществляется путем выбора из списка необходимого числа аргументов, что позволяет получать различнье модели, отражающие зависимости конкретных загрязнителей атмосферы от переменного числа аргументов. Представлена программная часть модели, состоящзая из набора программных модулей для проведения мониторинга, моделирования и прогнозирования. Приведень объектная модель программно-инструментальной системь для проведения мониторинга, моделирования и прогнозирования атмосферного загрязнениями алгоритм работь программы. Исходными данными программно-инструментальной системь для проведения мониторинга, моделирования и прогнозирования атмосферного загрязнения являются: список всех параметров, измерения которых проводились во время мониторинга, которые разделяются на две группы, а именно, загрязнителей атмосферы и метеорологических факторов; список всех мест проведения измерений; начальная и конечная даты мониторинга. Вывод результатов работы программно-инструментальной системы для проведения мониторинга, моделирования и прогнозирования атмосферного загрязнения осуществляется в табличном и графическом режимах, в том числе, в виде электронной картыл. По результатам мониторинга построена электронная карта загрязнения атмосферы г. Одессы и г. Баку.

Annotation: The article describes a new program for automating the processing of monitoring data, modeling and forecasting atmospheric pollution. A model of a monitoring system for atmospheric pollution is proposed, which allows us to express the dependence of the concentration of a selected atmospheric pollutant on a set of meteorological factors. The model is based on the method of group accounting of arguments, which has proven itself in multi-factor modeling. The construction of the model is carried out by choosing from the list the required number of arguments, which makes it possible to obtain various models reflecting the dependence of specific air pollutants on a variable number of arguments. The software part of the model is presented, consisting of a set of software modules for monitoring, modeling and forecasting. An object model of a software and instrumental system for monitoring, modeling and forecasting atmospheric pollution with an algorithm for program operation is presented. The initial data of the software-instrumental system for monitoring, modeling and forecasting atmospheric pollution are: a list of all parameters measured during monitoring, which are divided into two groups, namely, atmospheric pollutants and meteorological factors; a list of all measurement sites; start and end date of monitoring. The output of the work of the software-instrumental system for monitoring, modeling and forecasting atmospheric pollution is carried out in tabular and graphical modes, including in the form of an electronic map. According to the results of the monitoring, an electronic map of air pollution in the city of Odessa and Baku was constructed.

Ключевые слова: загрязнение атмосферы, мониторинг, моделирование, прогнозирование, электронная карта, объектная модель.

Key words: atmospheric pollution, monitoring, modeling, prognostication, e-map, object model. 


\section{Система мониторинга загрязнение атмосферного воздуха}

Глобальные изменения климата, происходящие в наше время, являются одной из основных проблем, которые нужны решить человечеству. Загрязнение атмосферы как одна из главных причин этих изменений, напрямую влияет на качество среды обитания.Вся жизнь на планете зависит от состояния воздуха, которым мы дышим каждый день. Атмосферный воздух является жизненно важным компонентом окружающей природной среды, неотъемлемой частью среды обитания человека, растений и животных. Именно поэтому одной из наиболее важных экологических проблем Украины и всего мира является загрязнение атмосферного воздуха. Задача поиска новых методов прогнозирования загрязнения атмосферного воздуха и создание на их основе программных продуктов нового поколения является актуальной задачей мониторинга окружающей среды. В Национальном аэрокосмическом агентствег. Баку была разработана программно-инструментальная система для проведения мониторинга, моделирования и прогнозирования атмосферного загрязнения Апшеронского полуострова [1]. Программная часть системы включает набор программ для проведения мониторинга, моделирования и прогнозирования, а также для вывода результатов в табличном и графическом режимах, в том числе, в виде электронной карты. Предложенная модель атмосферного загрязнения выражает зависимость концентрации выбранного загрязнителя атмосферы от совокупности метеорологических факторов. Испытания программы показали высокую точность моделирования (около 5\%) и прогнозирования (менее 10\%)[2]. Однако система имела ряд недостатков. Все разработанные программы функционировали отдельно друг от друга и не имели общего графического интерфейса. Также, для построения моделей различных загрязнителей атмосферы применялся один и тот же набор влияющих параметров, что ограничивало гибкость моделирования. Все вышесказанное говорило о необходимости совершенствования данной системы.

Цель статьи: Представить усовершенствованную программно-инструментальная систему для проведения мониторинга, моделирования и прогнозирования атмосферного загрязнения.

\section{Программно-инструментальная система для проведения мониторинга, моделирования и прогнозирования атмосферного загрязнения}

В результате проведенной модернизации отдельные программы получили возможность вызова из главной формы, появилось единое рабочее пространство, система была дополнена новыми функциями. Новая программа написана в среде MATLAB с использованием объектно-ориентированного программирования. Поэтому для описания ее функций удобно показать схему программы, состоящей из отдельных программных модулей, условно называемых объектами. Каждый из этих объектов предназначен для выполнения своего определенного набора функций. Объекты взаимодействуют друг с другом и главной формой (рис. 1).

Работа программы управляется ее главной формой (MainForm). После подключения к базе данных мониторинга (MonitoringDatabase) программа в ответ получает следующую информацию:

- $\quad$ список всех параметров, измерения которых проводились во время мониторинга, которые разделяются на две группы, а именно, загрязнителей атмосферы и метеорологических факторов;

- список всех мест проведения измерений;

- начальную и конечную даты мониторинга.

Оператор выбирает из списка интересующий загрязнитель, отмечает один или несколько пунктов проведения мониторинга, задает начальную и конечную дату измерений. На основе введенных значений объект программы TQuery генерирует запрос к базе данных. Значения измерений, удовлетворяющие вышеуказанным критериям, передаются в объект TPreprocessor для предварительной обработки, включающей усреднение данных по заданным временным отрезкам, к примеру, по месяцам или годам. После предварительной обработки данные могут быть переданы в объект TProcessor для подготовки к выводу результатов, либо в объект ТМоdel для моделирования. Модель строится по методу группового учета аргументов, хорошо зарекомендовавшему себя при многофакторном моделировании. Для построения модели оператор выбирает из списка необходимое число аргументов. Таким образом, программа позволяет получать различные модели, отражающие зависимости конкретных загрязнителей атмосферы от переменного числа аргументов. Формула модели передается в объект TPrognosis для получения краткосрочного или среднесрочного прогноза. Результат прогнозирования передается в уже упоминавшийся выше объект ТРrocessor для подготовки к выводу. Вывод результатов мониторинга или прогноза может быть осуществлен в графическом или табличном виде. За вывод таблицы результатов отвечает объект ТТable. Выводятся усреднённые за выбранный период времени данные по всем пунктам измерений, а также в среднем по всей области проведения мониторинга. Объект ТМар предназначен для вывода результатов в виде электронной карты, состоящей из трех слоев. Первый слой представляет собой карту местности мониторинга. На втором слое кружками отмечены все пункты проведения измерений. На третьем слое выводится распределение концентрации выбранного загрязнителя атмосферы, построенное с помощью метода триангуляции Делоне. 


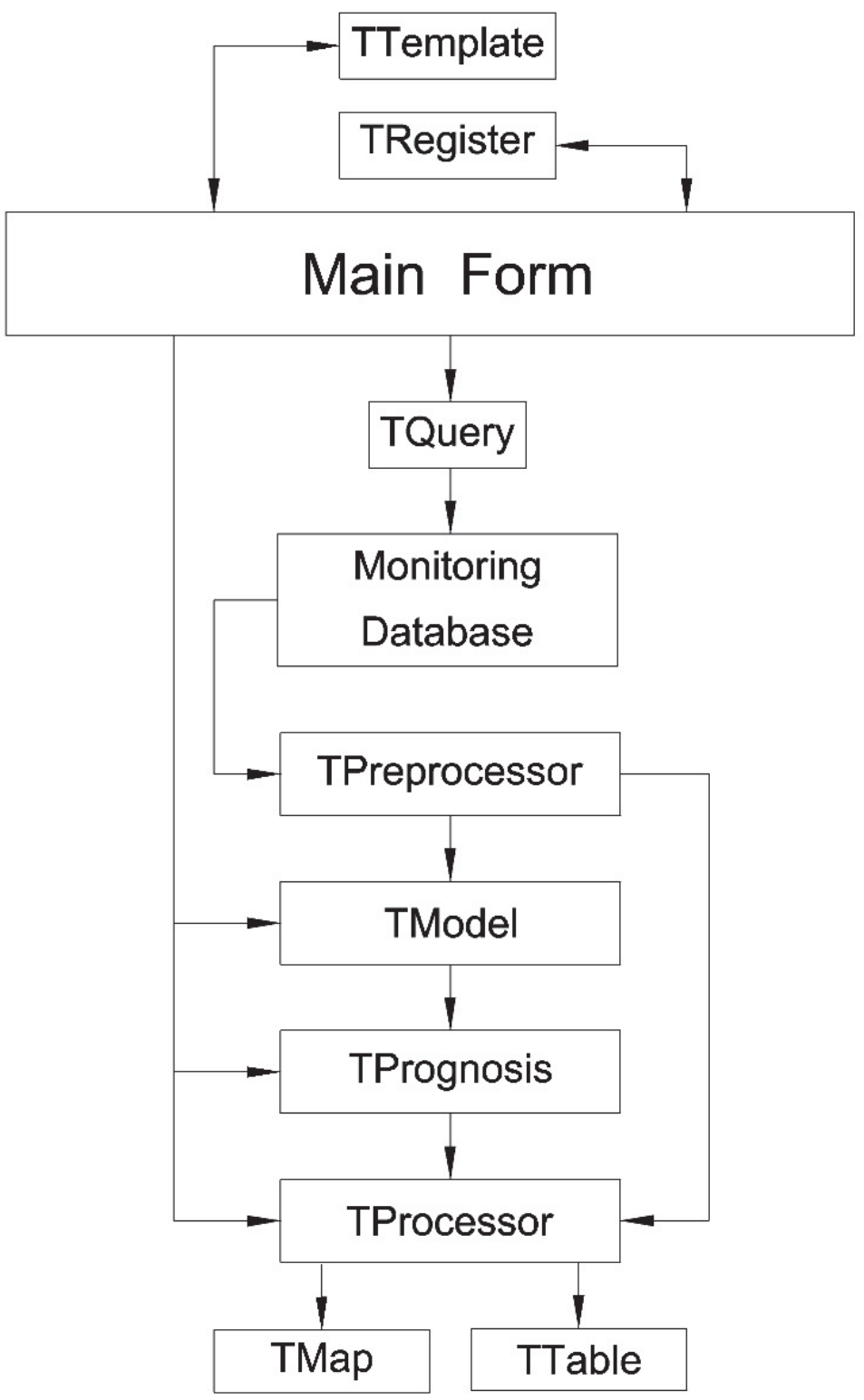

Рис. 1 - Объектная модель программы

Как указывалось, выше, программа позволяет моделировать зависимости концентраций различных загрязнителей атмосферы от разных метеорологических факторов. Чтобы каждый раз заново не выбирать, какие параметры использовать в качестве аргументов, настройки моделей можно сохранять в шаблонах. Для этого предназначен объект TTemplate. Файл шаблона может включать информацию о загрязнителе, учитываемых при моделировании метеорологических факторах, пунктах и датах наблюдений, типе модели и прогноза. Тип модели указывает на то, какую предварительную статистическую обработку информации из базы данных мониторинга следует производить. Тип прогноза указывает на краткосрочные (день, неделя, месяц) или среднесрочные (полугодие, год) прогнозы. Если в шаблоне какая-то информация отсутствует, принимаются значения по умолчанию. Информация в шаблоне записывается в виде тегов и их значений.

Наконец, объект TRegister служит для взаимодействия с реестром Windows, в котором хранятся настройки программы по умолчанию. С помощью реестра можно открывать новый рабочий проект с настройками из шаблона по умолчанию, последний использованный проект или любой другой существующий проект.

Всю вышеописанную процедуру получения выборки, построения модели и прогноза, а также представления результатов мониторинга или прогнозирования можно представить в виде алгоритма, который приведен на рис. 2. 


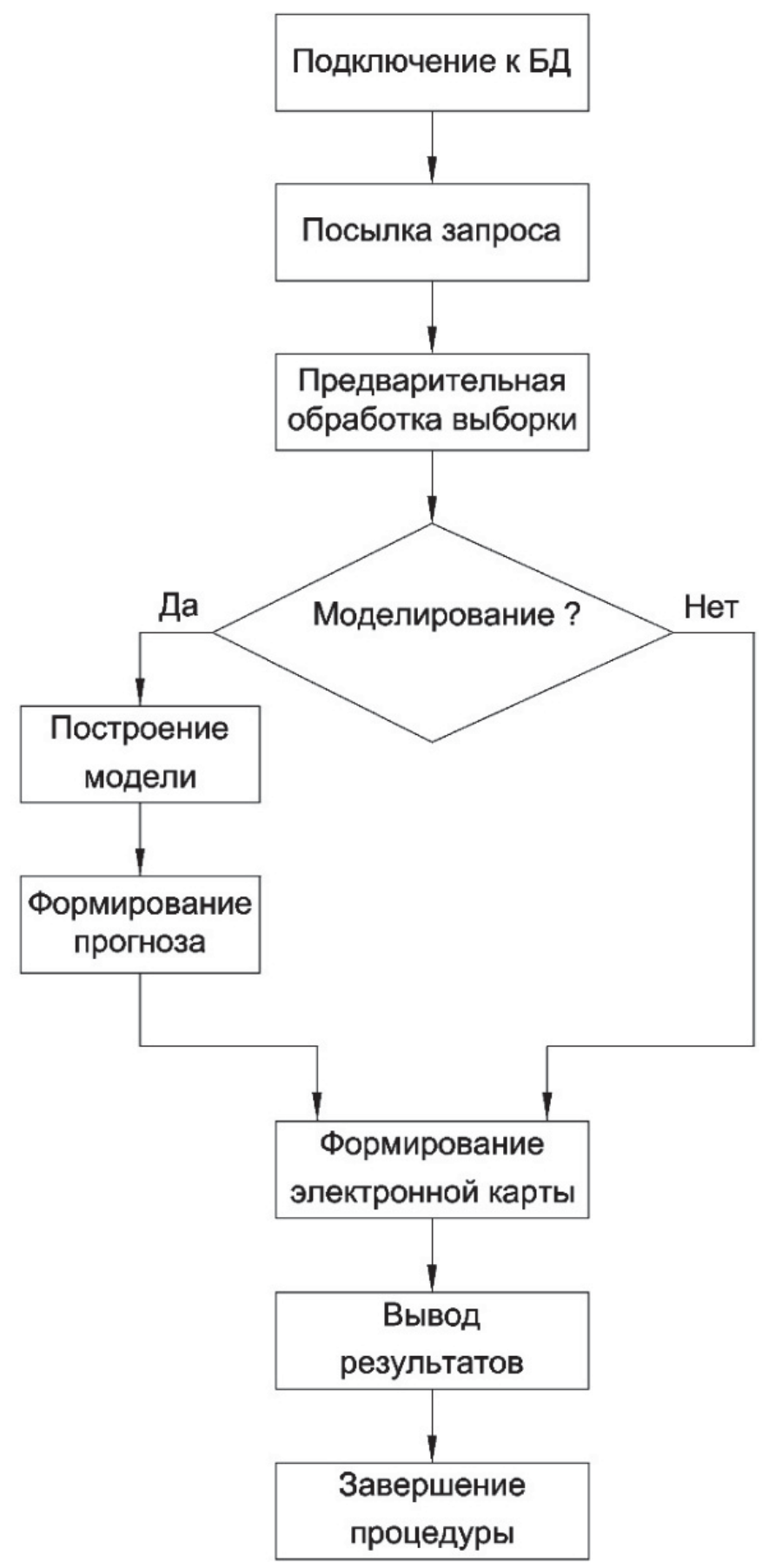

Рис. 2 - Алгоритм процедуры обработки данных мониторинга атмосферного загрязнения

Графический интерфейс программы представляет собой главную форму с несколькими панелями для формирования запроса, вывода табличных результатов и электронной карты, а также вспомогательные окна для моделирования, прогнозирования и выбора параметров. На рис. 3 приведена электронная карта, показывающая распределение максимальной концентрации в атмосфере загрязняющего вещества 4-х оксид азота по результатам мониторинга, проведенного в г. Баку в 2018 г. 

http://www.atbp.onaft.edu.ua/

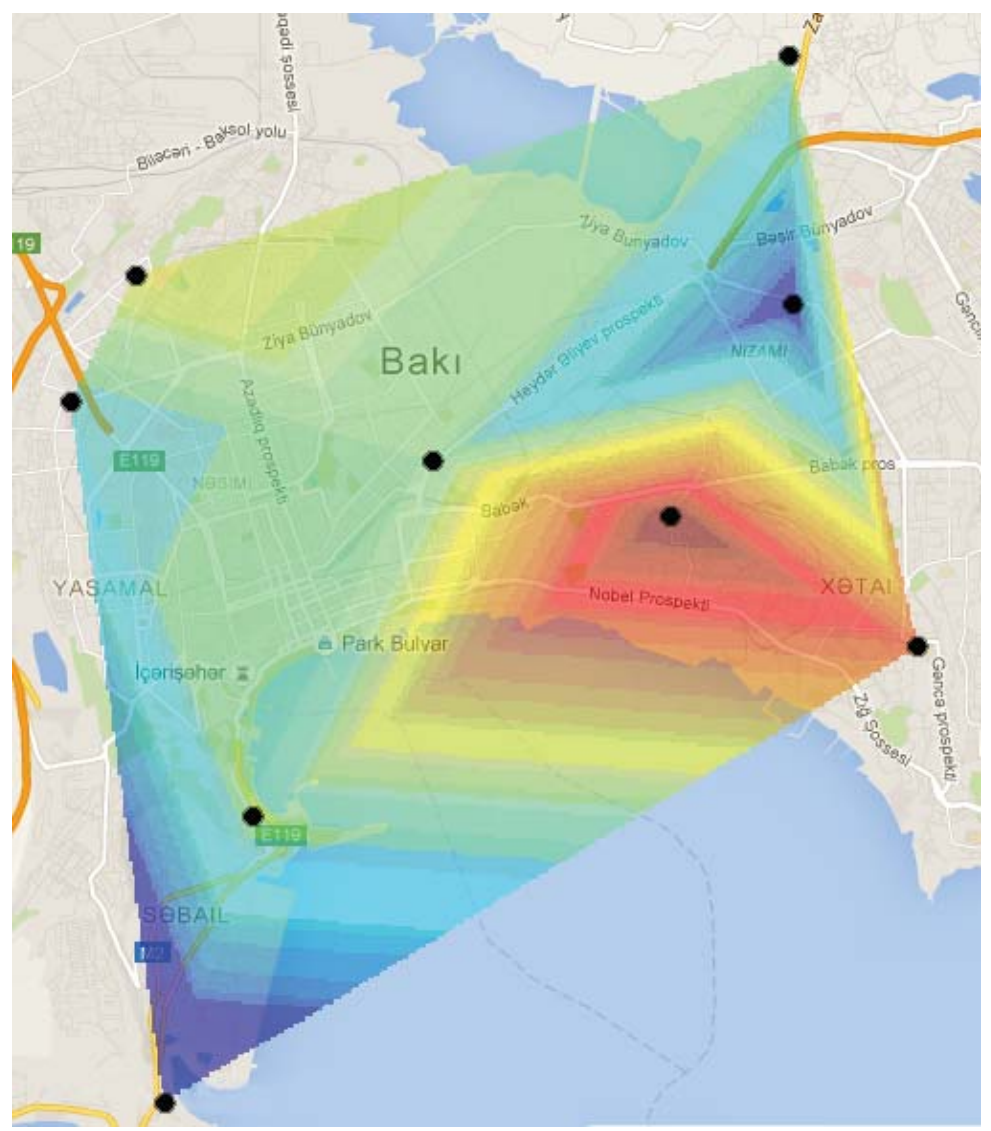

Рис. 3 - Распределение максимальных годовых значений Азот-4-оксида по г. Баку

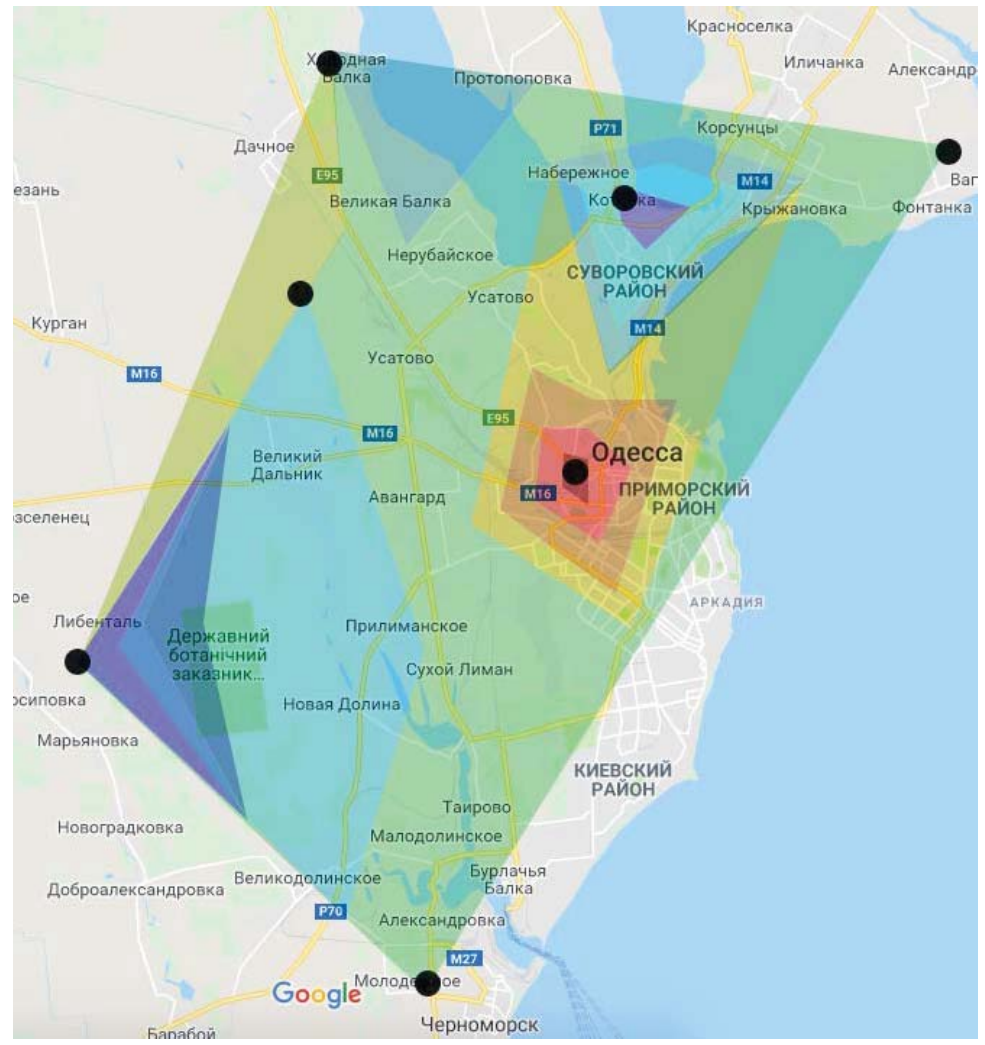

Рис. 4 - Распределение максимальных годовых значений Азот-4-оксида по г. Одесса 


\section{Заключение}

Таким образом, разработанная программа подтвердила свою работоспособность, удобство в работе, высокую точность моделирования и прогнозирования, гибкость в выборе аргументов для моделирования и наглядность представления результатов.

\section{Литература}

[1] Сулейманов Т.И. Обработка данных мониторинга атмосферных загрязнений города Баку / Т.И. Сулейманов, Н.Х. Мустафазаде, Р.К. Гулузаде //Приборы и системы. Управление, контроль, диагностика - Москва. - 2016. №10. -С. 41-45.

[2] Мамедов М.И. Применение метода группового учета аргументов для обработки данных мониторинга загрязнения атмосферы / М.И. Мамедов, Р.К. Гулузаде, Н.Х. Мустафазаде // Известия Азербайджанского Национального Аэрокосмического Агентства. -Баку. - 2015. - том 18. - №3. - С. 40-44.

\section{Referens}

[1] SuleymanovT.I., Mustafazade, N.H.,Huluzade R.K., Processing of monitoring data on atmospheric pollution in Baku. "Instruments and systems. Management, control, diagnostics” №10 (2016): 41-45.

[2] Mamedov, M.I.,Mustafazade, N.H., Huluzade R.K., Application of the group-based argument method for processing air pollution monitoring data. News of the Azerbaijan National Aerospace Agency №3 (2015): 40-44. 